Mens

revue d'histoire intellectuelle de l'Amérique française

\title{
Commémoration et idéologie nationale en Acadie. Les fêtes du bicentenaire de la Déportation acadienne
}

\section{Sacha Richard}

Volume 3, numéro 1, automne 2002

URI : https://id.erudit.org/iderudit/1024618ar

DOI : https://doi.org/10.7202/1024618ar

Aller au sommaire du numéro

Éditeur(s)

Centre de recherche en civilisation canadienne-française

ISSN

1492-8647 (imprimé)

1927-9299 (numérique)

Découvrir la revue

Citer cet article

Richard, S. (2002). Commémoration et idéologie nationale en Acadie. Les fêtes du bicentenaire de la Déportation acadienne. Mens, 3(1), 27-59.

https://doi.org/10.7202/1024618ar
Résumé de l'article

En 1955, le peuple acadien organisa de grandioses célébrations afin de souligner le deuxième centenaire d'un événement fondateur de l'identité et de l'histoire acadienne, la Déportation. Les " fêtes du bicentenaire acadien ", qu'on qualifia par la suite d'onzième congrès national acadien, attirèrent des milliers d'Acadiens et d'Acadiennes provenant de divers coins de l'Amérique. Le ralliement prit l'allure d'une grande célébration de la survivance acadienne, et ce faisant, véhicula l'idéologie traditionnelle de la survivance développée lors des premiers congrès nationaux acadiens de la fin du XIX ${ }^{\mathrm{e}}$ siècle. L'hypothèse avancée ici est que la commémoration du bicentenaire de la Déportation fournit une circonstance favorable pour réitérer l'importance de l'idéologie traditionnelle qui, selon les dirigeants, avait permis la survivance de la communauté acadienne et devait assurer son progrès dans l'avenir. 


\title{
COMMÉMORATION ET IDÉOLOGIE NATIONALE EN ACADIE.
} LES FÊTES DU BICENTENAIRE DE LA DÉPORTATION ACADIENNE ${ }^{1}$

\author{
Sacha Richard \\ Département d'histoire \\ Université d'Ottawa
}

\section{Résumé :}

En 1955, le peuple acadien organisa de grandioses célébrations afin de souligner le deuxième centenaire d'un événement fondateur de l'identité et de l'histoire acadienne, la Déportation. Les « fêtes du bicentenaire acadien ", qu'on qualifia par la suite d'onzième congrès national acadien, attirèrent des milliers d'Acadiens et d'Acadiennes provenant de divers coins de l'Amérique. Le ralliement prit l'allure d'une grande célébration de la survivance acadienne, et ce faisant, véhicula l'idéologie traditionnelle de la survivance développée lors des premiers congrès nationaux acadiens de la fin du XIX ${ }^{e}$ siècle. L'hypothèse avancée ici est que la commémoration du bicentenaire de la Déportation fournit une circonstance favorable pour réitérer l'importance de l'idéologie traditionnelle qui, selon les dirigeants, avait permis la survivance de la communauté acadienne et devait assurer son progrès dans l'avenir.

\section{Abstract :}

In 1955 the Acadian people organised a series of celebrations to commemorate the two bundredth anniversary of a key event in their history: the Acadian 
Expulsion. Later referred to as the Eleventh Acadian National Congress, les fêtes du bicentenaire acadien attracted thousands of Acadians from across the North American continent. The event quickly became a vast celebration of Acadian cultural survival, and in doing so, became an effective vebicle for the promotion of traditional Acadian nationalism, an ideology whose origins can be traced back to the first Acadian congresses of the late nineteenth century. This article argues that the commemoration of the expulsion's bicentennial offered an excellent occasion for Acadia's elites to reaffirm the importance of the traditional values that had ensured la survivance acadienne and would guarantee the future progress of the Acadian nation.

Celui qui lit l'bistoire du Grand Dérangement de 1755 sans lire le récit du Grand Rassemblement [a]cadien de 1955 est comme celui qui s'arrête à la Passion du Christ sans aller jusqu'au récit de Sa Résurrection glorieuse. ( $\mathrm{M}^{\mathrm{gr}}$ Albert Leménager, évêque de Yarmouth,1954)

En août 1955, le peuple acadien était en liesse. Il soulignait le bicentenaire d'un événement marquant de son histoire - la Déportation de $1755^{2}$ - par de grandioses manifestations religieuses et patriotiques qui eurent lieu au NouveauBrunswick et en Nouvelle-Écosse. Le programme des fêtes du bicentenaire acadien, qui comprenait une reconstitution historique, un festival de folklore, des messes pontificales, un défilé de chars allégoriques, un concert d'artistes acadiens, des banquets, des feux d'artifice et bien d'autres activités, témoignait de l'importance attachée à cet événement. Il ne s'était jamais auparavant organisé des célébrations d'une telle envergure en Acadie et leur succès avait été grandement anticipé. Les hommes à qui on avait confié la tâche de les mettre sur pied avaient prédit que les fêtes du bicentenaire constitueraient un événement " sans précédent dans notre histoire $3^{3}$ » 
Le ralliement fut d'une telle importance qu'il fut hissé, après coup, au rang des grands congrès nationaux acadiens ${ }^{4}$.

L'organisation du rassemblement de 1955 marquait ainsi la relance d'une tradition acadienne importante, celle du « congrès national ». Ces grands ralliements patriotiques, qui avaient lieu de façon sporadique entre 1881 et 1937, étaient un symbole puissant de la survivance, une manifestation de la vitalité et du progrès de la collectivité acadienne ${ }^{5}$. Mais surtout, les premiers congrès avaient permis la définition d'une idéologie nationale. C'est du moins l'opinion du sociologue $\mathrm{Ca}$ mille-Antoine Richard, qui a étudié le premier congrès national acadien tenu en 1881 à Memramcook. Richard a constaté que l'élite acadienne a profité du rassemblement pour formuler et diffuser une première idéologie nationale, par la suite connue comme "l'idéologie traditionnelle de la survivance", fondée sur le respect de l'histoire, de la religion, et des ancêtres $^{6}$. Au cours de cette étude, nous entendons réévaluer la place du ralliement de 1955 dans l'évolution de cette idéologie. Nous verrons que les fêtes du bicentenaire, qui célébrèrent la survivance acadienne, véhiculèrent le discours idéologique traditionnel.

Les années cinquante en Acadie sont encore très peu étudiées et, par conséquent, le ralliement de 1955 n'a fait l'objet d'aucune étude approfondie? ${ }^{7}$ Le sociologue Jean-Paul Hautecœur consacre quelques pages de son ouvrage L'Acadie $d u$ discours à une brève analyse du message des célébrations du bicentenaire. Hautecœur estime que les fêtes ont symbolisé un « rite de passage », un «temps libre entre deux phases » où l'élite acadienne inaugura une "ère nouvelle ${ }^{8} »$. Il soutient ainsi que les fêtes marquèrent le subtil début de la période moderne de l'Acadie. De son côté, Philippe Eddie est plutôt enclin à " limiter le caractère novateur de l'ère ouverte " par les fêtes du bicentenaire puisqu'il croit que ces célébrations, 
qui mirent en valeur le nationalisme traditionnel de la survivance, ne constituèrent pas un tournant idéologique important'. La prédominance de l'idéologie traditionnelle dans le discours des fêtes nous laisse également croire que les célébrations s'inscrivirent en continuité avec la situation antérieure. Nous ne sommes toutefois pas décidé à considérer les fêtes de 1955 comme insignifiantes simplement parce qu'elles récupérèrent le discours idéologique traditionnel.

Les analyses des fêtes commémoratives en Amérique ne cessent de croître depuis une vingtaine d'années. De nombreux chercheurs, influencés par les courants idéologiques postmodernes, tentent de comprendre le processus par lequel une société conceptualise, communique et réinvente l'histoire $^{10}$. Leurs études démontrent que la commémoration se répercute sur la (re)création d'identités collectives, car elle redéfinit la relation d'une collectivité à son passé. Cette observation s'avère particulièrement intéressante puisque les fêtes commémoratives du bicentenaire de la Déportation s'insèrent $a$ priori dans un contexte de transition au Canada français. Des sociologues et des historiens ont écrit que l'identité canadienne-française traditionnelle fut profondément remise en question au cours de l'après-guerre ${ }^{11}$. Évidemment, cette mutation de l'identité canadienne-française eut de profondes répercussions sur les communautés françaises minoritaires, comme le confirment certaines études récentes. Marcel Martel constate que le renouvellement du discours identitaire au Québec dans les années cinquante annonçait la déchirure du Canada français, qui survint lors de Révolution tranquille $^{12}$. La transformation du Canada français obligea par la suite les communautés françaises hors Québec à redéfinir leur identité nationale. L'Acadie ne fit pas exception à cette règle ${ }^{13}$. 
Les fêtes du bicentenaire se prêtent à une étude discursive intéressante. Une analyse approfondie de diverses sources, tels les articles de journaux, les discours, la correspondance et les procès-verbaux, nous permet de saisir l'importance constante de l'idéologie traditionnelle en Acadie qui trouva son expression dans les fêtes commémorant le bicentenaire de la Déportation. Le rassemblement de 1955 fut l'occasion de célébrer la survivance et, du même coup, de réaffirmer la valeur de l'idéologie qui avait assuré la survie et le progrès de la société acadienne et devait encore l'assurer dans l'avenir selon l'élite. Cette étude tâche, en premier lieu, de présenter les raisons qui motivèrent l'élite acadienne à souligner le bicentenaire de la Déportation et l'importance de cet événement dans le récit national acadien. Puis, nous procédons à une analyse détaillée du discours véhiculé lors des célébrations pour y dégager les thèmes dominants. Enfin, nous verrons comment l'élite acadienne eut recours au passé pour préparer l'avenir.

\section{«On peut le dire tout de suite : il ne s'est jamais organisé pareilles fêtes dans les Provinces Maritimes ${ }^{14}$ "}

Le centenaire de la Déportation était passé sous silence en Acadie. La situation socio-économique des Acadiens, qui étaient dépourvus de ressources financières, d'institutions et de chefs, n'avait guère favorisé l'organisation de célébrations. Cependant, la dernière moitié du XIX ${ }^{e}$ siècle avait été témoin d'un prodigieux essor démographique, politique et culturelle communément appelé la « Renaissance acadienne ». Pendant cette période, la population acadienne et son poids démographique avaient augmenté de façon notable et sa participation s'était accrue au sein des partis politiques. La communauté avait surtout solidifié son armature par la création de nou- 
veaux collèges, de journaux, d'institutions nationales, de porteparole et de symboles collectifs ${ }^{15}$. Les jalons marquants de la Renaissance acadienne furent la fondation du collège SaintJoseph de Memramcook en 1864, le lancement du journal Le Moniteur Acadien en 1867, la création du collège Saint-Louis (Edmundston) en 1874, la tenue des premiers congrès nationaux acadiens en 1881 et 1884 qui donnèrent lieu à la fondation de la Société nationale l'Assomption et à l'adoption des symboles nationaux que sont la fête nationale du 15 août, une sainte patronne, un hymne (Ave Maris Stella) et un drapeau (le tricolore française rehaussé d'une étoile dorée dans la bande gauche) et enfin la fondation du quotidien L'Évangéline $e^{16}$ en 1887 . Le progrès accompli depuis cette époque avait été constant, si bien qu'au mitan du $\mathrm{XX}^{\mathrm{e}}$ siècle, les Acadiens étaient prêts à célébrer un événement marquant de leur histoire.

Le projet de commémoration de la Déportation fut placé sous les auspices de l'organisation jugée la plus compétente pour l'exécuter, soit la Société nationale l'Assomption (SNA). La Société, fondée en 1881 lors du premier congrès national acadien de Memramcook, regroupait des délégués de toutes les régions où vivaient des Acadiens. Elle se considérait comme le porte-parole officiel de cette communauté ${ }^{17}$. Malgré des débuts impressionnants, elle était tombée dans une période d'inactivité après le dernier congrès tenu en 1937. À l'hiver de 1950, d'anciens membres du conseil de direction de la Société, réunis pour étudier diverses questions d'intérêt national, exprimèrent le désir de jeter les bases d'un organisme qui assurerait le développement du peuple acadien. Ces hommes croyaient que le bicentenaire, qui aurait lieu cinq ans plus tard, fournirait une occasion idéale pour rallier le peuple, ranimer la conscience nationale acadienne et donner un " regain de vie » à la Société par la création d'un secrétariat per- 
manent ${ }^{18}$. Le comité spécifia que, lorsque le peuple verrait le travail de la Société, il comprendrait l'importance de la raviver et de soutenir son développement. En somme, un des objectifs avoués des célébrations du bicentenaire de la Déportation était de relancer les activités de la SNA et ainsi de doter l'Acadie d'un organisme national militant qui aurait pour fonction de stimuler le nationalisme acadien, d'amener la population à prendre conscience de l'importance de l'épanouissement de l'Acadie et à agir en conséquence ${ }^{19}$.

À part ce motif, le conseil jugea que le bicentenaire de la Déportation méritait d'être souligné en raison de son importance passée et à venir. Lors de la première réunion tenue à l'hiver 1950, le conseil de direction de la Société décida de commémorer le deuxième centenaire de la Déportation avec «beaucoup d'éclat » puisqu'il était d'avis qu'un " événement tel que celui du Grand Dérangement ne doit point être oublié à cause des salutaires leçons qui s'en dégagent pour l'avancement du peuple martyr ${ }^{20} \gg$. Le président de la SNA, le Dr Georges Dumont, précisa par la suite que « [c]e triste événement a eu trop de retentissement dans la vie de notre peuple pour que son anniversaire ne soit pas marqué par des grandes fêtes patriotiques et religieuses ${ }^{21} »$. Puisque son souvenir constitue l'un des piliers de l'histoire et de l'identité acadienne, il était nécessaire que la Déportation de 1755 soit commémorée d'une manière qui fasse honneur à son importance à l'occasion de son bicentenaire.

La Société n'étant pas en mesure d'organiser des célébrations d'une telle envergure, ses dirigeants créèrent un comité qui allait agir en son nom. Créé en 1953 dans la région de Moncton, le " comité central des fêtes » devait maintenir un " contact étroit avec les autorités religieuses et les dirigeants nationaux ${ }^{22} »$. Son président, le sénateur Calixte Savoie, s'entoura de membres importants de la société acadienne, 
notamment le Père Clément Cormier, recteur de l'Université Saint-Joseph, Emery LeBlanc, directeur-gérant de L'Évangéline, Gilbert Finn, gérant général adjoint de la Société Mutuelle l'Assomption, le juge Adrien Cormier, Gilbert Robichaud, chef de service à la Société l'Assomption, Euclide Daigle, rédacteur en chef de L'Évangéline. Peu de temps après, on confia l'organisation proprement dite des fêtes à un avocat réputé de la région, Adélard Savoie ${ }^{23}$. L'organisation des fêtes, qui fut prise en main par les dirigeants religieux, politiques et professionnels de l'Acadie, revêtit évidemment un caractère élitiste.

Le comité organisateur prit dès lors la décision de prolonger la commémoration de la Déportation sur une année complète afin de permettre à tous les Acadiens de la diaspora d'y prendre part. Des fêtes régionales eurent lieu dans plusieurs localités des provinces maritimes, du Québec, des ÉtatsUnis, de l'Ontario et de l'Ouest canadien ${ }^{24}$. L'esprit des fêtes atteignit son point culminant lors de la "grande apothéose " des célébrations du bicentenaire, qui se tint du 8 au 16 août 1955. Le comité organisateur avait décidé de ne rien laisser au hasard pour cette grande finale ${ }^{25}$. Il rehaussa le prestige des manifestations religieuses et patriotiques en lançant des invitations à plusieurs personnalités. L'impressionnante liste des invités comportait nombre de dignitaires religieux et civils venus du reste du Canada, des États-Unis et de l'Europe ${ }^{26}$. De plus, le comité de publicité, dont le mandat était « de voir à ce que les fêtes de 1955 gardent la vedette jusqu'à la fin ", employa tous les moyens à sa disposition pour s'assurer que ces dernières demeurassent sous les feux de la rampe $e^{27}$.

Enfin, le comité décida que les fêtes nationales du mois d'août ne commémoreraient pas uniquement la Déportation, mais plutôt les quatre centenaires de l'histoire acadienne. L'épanouissement du peuple acadien depuis la Déportation 
fut célébré à Moncton le 11 août sous le thème "L'Acadie triomphante » ou «L'Acadie de 1955 ». L'ambiance des fêtes à Moncton en fut une de jubilation, car on célébra les réalisations du peuple acadien dans tous les domaines ainsi que les organisations et les institutions ayant assuré sa survivance. Le 12 août, on se déplaça à Memramcook pour souligner le centenaire de la relève acadienne. Sous le thème «L'Acadie à l'aurore de la renaissance » ou "L'Acadie de 1855 ». On y organisa des célébrations visant à honorer les rescapés de la Déportation et à souligner les premières velléités de survivance en Acadie. Les fêtes atteignirent leur apogée le 15 août à Grand-Pré, où on souligna le bicentenaire de la Déportation ou «L'Acadie de 1755 » en rappelant l'ascension du peuple acadien. La quatrième fête, célébrée à Port-Royal le 16 août, souligna le $350^{e}$ anniversaire des débuts de la vie française en Amérique du Nord par des célébrations rappelant l'établissement des Français à Port-Royal mené par Champlain, De Monts et Poutrincourt ${ }^{28}$.

\section{"C'est bien le miracle acadien qu'il s'agit de célébrer ${ }^{29}$ "}

On ne saurait nier que ce fut la survivance acadienne qui fut à l'honneur en 1955. Les fêtes du bicentenaire visèrent à souligner la Déportation pour évoquer, avant tout, le progrès du peuple acadien. C'est ce qu'expliqua Adélard Savoie lors d'un discours explicitant l'objectif des célébrations :

Non, les fêtes du bicentenaire ne seront pas une invitation aux souvenirs amers ni aux querelles du passé. Elles seront plutôt un splendide tribut d'action de grâces et de reconnaissance. Elles seront un hommage filial et sincère à la mémoire de nos pères et aux vertus qu'ils ont incarnées. Elles seront aussi une grandiose manifestation du fait français en terre d'Acadie $[. . .]^{30}$. 
Ainsi, les célébrations commémorant le bicentenaire d'un triste événement prirent l'allure de grandes festivités de la survivance dans lesquelles l'idéologie traditionnelle acadienne s'intégra facilement. À l'instar de ce qui s'était fait dans les congrès nationaux précédents, les fêtes de 1955 reprirent le discours idéologique de la survivance mettant en valeur la bonne entente ethnique, l'importance de la Renaissance acadienne, la foi catholique ainsi que l'histoire et les traditions.

Le comité organisateur avait dû prendre rapidement conscience que cette commémoration d'un des moments les plus difficiles de la cohabitation entre Français et Anglais dans les Maritimes risquait de faire surgir des conflits ethniques ou linguistiques. En 1953, le conseil de direction de la Société avait d'ailleurs noté son désir de maintenir la politique de bonne entente en mettant en valeur " les arguments qui blesser[aient] le moins les Canadiens d'autre[s] langue[s] et susciter[aient] l'enthousiasme des nôtres ${ }^{31} »$. Le discours de la bonne entente imprégna conséquemment la conception, l'organisation et la programmation des célébrations. Le comité organisateur répéta avec insistance que les fêtes du bicentenaire susciteraient des " sentiment positifs » et ne réveilleraient pas de vieilles animosités. L'élite religieuse, souhaitant à tout prix conférer un caractère chrétien aux célébrations, déclara à l'unisson que le peuple acadien n'avait " aucune intention de ressasser dans [son] cœur aigri les vieilles rancunes et y rouler de sombres desseins de haine et de vengeance ${ }^{32} »$. De nombreux politiciens acadiens, actifs tant à Fredericton qu'à Ottawa, abondèrent également dans le même sens ${ }^{33}$.

Sans nul doute, ce fut le geste d'amitié envers la reine qui symbolisa le mieux cette volonté d'harmonie ${ }^{34}$. Par un geste tout à fait imprévisible, et quelque peu incongru étant donné la nature de la commémoration, l'élite acadienne décida de 
lancer une invitation à Sa Majesté Élisabeth II la conviant à prendre part aux célébrations qui commémoreraient, non pas la Déportation, mais plutôt la survivance acadienne. Souhaitant démontrer leur « gratitude envers les personnes et les institutions qui ont contribué de quelque façon à la Renaissance du peuple acadien ", les organisateurs exprimèrent la reconnaissance du peuple acadien pour avoir trouvé, sous la Couronne d'Angleterre, "les conditions qui leur ont permis de réaliser les progrès contemporains qui font l'objet de leurs réjouissances d'aujourd'hui ${ }^{35}$ ». Le comité organisateur termina son message en proclamant les « sentiments d'affection et de loyauté » des Acadiens à l'égard de la Couronne ${ }^{36}$. En favorisant un esprit de paix, le comité organisateur escomptait que les fêtes seraient une célébration, plutôt qu'une réédition, d'un passé tragique ${ }^{37}$.

Ainsi, pour s'assurer de ne pas perturber l'équilibre précaire entre les populations française et anglaise des provinces maritimes, mais surtout pour maintenir la population dans une atmosphère de réjouissance, les organisateurs s'assurèrent que la commémoration de la Déportation allât de pair avec une célébration de la survivance. Hector Robichaud, député fédéral de la circonscription de Gloucester, exprima bien cette intention lorsqu'il déclara à ses collègues à la Chambre des communes que les Acadiens n'avaient pas

l'intention de raviver certains ressentiments nés d'une triste et malheureuse époque de l'histoire de notre peuple, mais plutôt de marquer officiellement et solennellement la renaissance et la survivance d'un petit groupe qui, voilà exactement deux siècles, était voué à la dispersion et à un anéantissement presque assurée ${ }^{38}$.

Bien que l'événement commémoré ait constitué la grande épreuve du peuple acadien et l'un des moments les plus difficiles de son histoire, le comité désirait que les fêtes 
reflètent « la joie profonde d'une résurrection plutôt que la tristesse d'un Libera ${ }^{39}$ ». Calixte Savoie, clamant que « ceux que l'on croyait morts sont encore bien vivants », précisa que c'était " précisément la renaissance de notre peuple, son épanouissement et son rayonnement que nous nous préparons à célébrer avec pompe et éclat ${ }^{40}$ ». Les fêtes mirent donc l'accent sur la survivance, et particulièrement sur l'importance de la relève du XIX ${ }^{\mathrm{e}}$ siècle.

L'interprétation historique traditionnelle accorda une grande valeur à la Renaissance acadienne. Calixte Savoie souligna son rôle intégral dans l'histoire de la survivance : « [c]'est l'Acadie de 1855 qui a vu le peuple acadien prendre conscience de son existence ${ }^{41} »$. L'élite se fit le chantre de la Renaissance acadienne non seulement par le discours, mais également par des manifestations populaires, comme lors du défilé historique qui eut lieu dans les rues de Moncton ayant pour thème «L'Acadie rayonnante ». Ce défilé était divisé en trois sections représentant les grandes étapes de l'histoire de l'Acadie : la première se voulait un rappel de la vie en Nouvelle-France, la deuxième illustrait l'histoire d'Évangéline et la troisième soulignait la période de la relève. Les organisateurs consacrèrent près de la moitié des chars allégoriques à cette dernière partie qui rendait hommage aux pionniers politiques, nationaux et religieux de l'Acadie ainsi qu'aux premières institutions nationales, éducatives et coopératives établies dans la deuxième moitié du XIX ${ }^{\mathrm{e}}$ siècle. Ces chars furent commandités par les institutions acadiennes nées à cette époque, telles que les universités Saint-Joseph et Sacré-Cœur, les journaux L'Évangéline et Le Madawaska, la Société mutuelle l'Assomption et la Société nationale l'Assomption.

Les organisateurs des fêtes célébrèrent aussi la période de la Renaissance acadienne par le biais d'une reconstitution historique, le pageant de l'Acadie, qui fut présentée sur six soi- 
rées et qui marqua un des points culminants des fêtes. Cette reconstitution historique, élaborée par le père Laurent Tremblay, directeur du Théâtre Chrétien du Québec, illustrait en vingt scènes plus de 300 ans d'histoire acadienne. La vie du peuple acadien avant la Déportation fut résumée en quatre scènes qui racontaient l'arrivée des premiers colons ainsi que la vie paisible en Acadie avant la Conquête anglaise de 1713. La Déportation fut présenté en cinq scènes qui traitaient de la tragédie comme telle, l'existence misérable des déportés aux États-Unis et du retour pénible de certains groupes d'exilés. Les onze dernières scènes étaient réservées à la vie acadienne après le retour de l'exil et, plus précisément, à la période de la Renaissance acadienne. Ces scènes relataient l'arrivée des missionnaires, la fondation des collèges acadiens et de la Société mutuelle l'Assomption, la tenue des grands congès acadiens, le travail des congrégations religieuses et l'essor dans le domaine culturel ${ }^{42}$.

Il importe également de noter que l'utilisation des symboles nationaux adoptés lors des premiers congrès nationaux contribua aussi à promouvoir l'importance de la Renaissance acadienne. Le tricolore acadien occupa une place de premier plan. L'Évangéline proclamait qu'on ne pouvait «abuser de l'usage du drapeau acadien à travers toute l'Acadie en ces jours de fêtes ${ }^{43} »$. Tout comme le drapeau, Évangéline, l'héroïne mystique du peuple acadien, occupa une place importante ${ }^{44}$. Dès 1953, on créa un comité des costumes chargé de confectionner des centaines de costumes d'Évangéline. À l'approche des fêtes, le quotidien L'Évangéline annonça que « la dame ou demoiselle qui portera[it] le costume d'Évangéline ou un autre costume de tradition acadienne sera[it] toujours à la mode ${ }^{45} »$. Le moment était également propice pour rendre hommage à Henry Wadsworth Longfellow, l'écrivain américain qui avait rendu le personnage d'Évangéline célèbre. Lors 
du dévoilement d'un monument en son honneur à Grand-Pré le 15 août, le professeur Willie Belliveau, de l'Université Sainte-Anne, souligna l'importance symbolique d'Évangéline, " le symbole, la figure mystique du peuple acadien », dans le récit national ${ }^{46}$.

La religion occupait également une place prédominante dans cette idéologie de la survivance et on ne pouvait guère penser à commémorer le progrès du peuple acadien sans rendre hommage à la " Providence ». L'élite laïque et religieuse souligna très tôt que les célébrations seraient d'abord des fêtes d'action de grâce, des manifestations extraordinaires par lesquelles on la remercierait pour la « résurrection » du peuple acadien ${ }^{47}$. Dans le message qui fut envoyé au souverain pontife, $\mathrm{M}^{\mathrm{gr}}$ Norbert Robichaud, archevêque de Moncton et porteparole de l'élite religieuse acadienne, écrivit qu'après la dispersion de 1755, les exilés avaient remis leur destin entre les mains de Dieu. Reconnaissants de la protection qu'Il leur avait accordée, les Acadiens avaient voulu organiser des fêtes dont " la note dominante sera[it] la reconnaissance à Dieu pour sa protection providentielle et l'imploration de nouvelles grâces pour leur permettre de rester fidèles à leur glorieux passé $e^{48} »$. Cette vision du passé reposait sur la notion d'une mission divine assignée par la Providence. Les Acadiens, pour qui la diffusion des valeurs chrétiennes et de la culture française constituait une vocation, avaient subi la dispersion pour ne pas dévier de cette mission ${ }^{49}$.

Il n'est donc pas étonnant de constater que les manifestations religieuses occupèrent une place de premier plan dans le programme des fêtes. L'ouverture officielle des célébrations, qui eut lieu devant la cathédrale Notre-Dame de l'Assomption à Moncton, fut soulignée par un geste de reconnaissance collectif. Le soir du 10 août, au son des cloches, " chaque famille se $\mathrm{m}[\mathrm{it}]$ à genoux, dehors, devant sa maison, et récit[a] 
à haute voix la belle prière du bicentenaire " dans laquelle on confiait à Dieu le sort du peuple acadien ${ }^{50}$. L'événement marquant des fêtes fut incontestablement la troisième messe pontificale, célébrée le 15 août à Grand-Pré par $\mathrm{M}^{\mathrm{gr}}$ Giovanni Panico, délégué apostolique au Canada. Environ 10000 Acadiens se déplacèrent pour prendre part à cette célébration qui réunissait trois archevêques, 11 évêques, 200 prêtres et 50 religieuses en provenance de la Nouvelle-Écosse, du Nouveau-Brunswick, du Québec, de Terre-Neuve, de l'Ouest canadien, et même de Paris et d'Haitt ${ }^{51}$. Assurément, la présence de ces dignitaires ecclésiastiques rehaussa le caractère religieux des célébrations.

L'idéologie traditionnelle était, somme toute, profondément arrimée au passé. L'élite ne pouvait trouver meilleur forum pour la promouvoir que lors des fêtes commémoratives. $\mathrm{M}^{\mathrm{gr}}$ Camille-André LeBlanc de Bathurst expliqua que le bicentenaire de la Déportation devait servir de leçon d'histoire à tous les Acadiens. À la veille de l'inauguration des célébrations, il déclara que cette année " n'[était] pas de trop pour étudier, réétudier notre histoire, la savourer, s'en imprégner, et s'en nourrir $[\ldots]^{52} »$. L'élite déplorait un manque de fidélité au passé, source de la survie nationale. $\mathrm{M}^{\mathrm{gr}}$ Leménager, évêque de Yarmouth, expliqua l'importance de renouer avec l'histoire, fondement de la survivance : "Les Acadiens ne $\mathrm{d}[$ oiv]ent pas perdre de vue cet événement unique de leur histoire, et peut-être de l'Histoire tout court, parce qu'il constitu[e] le pivot de leur existence nationale ${ }^{53} »$. L'élite saisit l'occasion en 1955 pour vanter l'attachement au passé qui, conformément au discours traditionnel, était considéré comme une condition sine qua non de la survive nationale acadienne.

En se rappelant le passé, les fêtes du bicentenaire parvinrent à rendre hommage aux vertus des aïeux. $\mathrm{M}^{\mathrm{gt}}$ Robichaud 
spécifia, quelques mois avant le début des célébrations, que celles-ci exalteraient leur mémoire :

Les fêtes de l'an prochain auront donc l'heureux résultat d'effectuer un retour vers nos origines, et permettront à nos compatriotes de réfléchir et de méditer sur la nécessité de rester fidèles aux traditions des ancêtres et de sauvegarder les valeurs spirituelles et culturelles qu'il nous ont léguées ${ }^{54}$.

Calixte Savoie proclama à son tour que le peuple acadien devait demeurer fidèle à l'exemple des ancêtres : «Ce qui a fait la force de nos ancêtres fera aussi la nôtre. Marchons sur leurs traces ${ }^{55} »$. Reconnaissant que le culte du passé avait été la source de la survivance acadienne et de sa ferveur nationale, l'élite prêcha la fidélité indéfectible aux vertus et aux qualités morales des ancêtres, notamment à leur courage, à leur persévérance, à leur foi catholique et à leur patriotisme. On profita de l'occasion pour décorer quatre Acadiens incarnant ces caractéristiques de la prestigieuse médaille de l'Ordre de la Fidélité française ${ }^{56}$.

L'idéologie traditionnelle reposait donc sur une vision du passé apte à raviver une conscience nationale que l'élite jugeait essentielle au maintien de la cause de la survivance. En commémorant un passé glorieux, le comité organisateur espérait instiller le sens de la fierté du passé à la jeunesse acadienne qui, croyait-on, oubliait parfois l'importance des traditions ancestrales. Cette commémoration devait inciter la jeune génération acadienne à continuer le travail entamé par ses ancêtres plus de deux cents ans auparavant. C'est ce que $\mathrm{M}^{\mathrm{gr}}$ Leménager avait en tête lorsqu'il déclara qu'une « incursion aussi consciencieuse dans un passé héroïque ne manquera[it] pas de décupler les énergies pour la survivance $^{57} \gg$. Dans le but d'assurer une relève, le Secrétariat des fêtes de 1955 publia également de nombreuses saynètes, des- 
tinées à un public d'écoliers, qui rendaient hommage aux chefs de file acadiens qui avaient su rester fidèles à « leur Dieu, à leur langue et à leur patrie ${ }^{58}$ ». L'élite espérait qu'un tel message amènerait la jeunesse, et la population acadienne en générale, à prendre conscience de ses devoirs et de ses responsabilités à l'égard de la nation et de son avenir. Le président général des fêtes résuma bien cette pensée lorsqu'il affirma que les fêtes visaient à " fouetter » la fierté nationale acadienne puisqu'il fallait " coûte que coûte entretenir vivante la flamme de notre vie nationale ${ }^{59} \%$.

\section{« En avant, Acadie ! ${ }^{60}$ »}

L'élite acadienne espérait donc commémorer le passé en vue d'y puiser de la force pour l'avenir. Tout en louant les vertus de l'idéologie de la survivance, elle exprima en même temps sa volonté de préparer l'avenir. Cette tâche s'avéra surtout importante puisque l'élite était persuadée que la société acadienne se trouvait à un carrefour important de son cheminement national et qu'elle se préparait à réaliser des exploits. À l'aube des fêtes, L'Évangéline pouvait écrire que le peuple acadien se trouvait au seuil d'un temps nouveau: «Nous avons tous l'impression - une impression appuyée sur des faits éclatants, sur les œuvres progressives et des possibilités raisonnables - que l'Acadie va vivre de nouveaux temps ${ }^{61} »$. Il importait ainsi de se rassembler afin de faire le bilan des réalisations acadiennes, mais surtout de préciser "le rôle que notre peuple dev[ait] jouer d'ici son Tricentenaire ${ }^{62} »$.

Cette volonté de se tourner vers l'avenir se dessine dans le mémoire envoyé au premier ministre du Québec quelques mois avant les fêtes. Le comité organisateur, qui demandait $25000 \$$ au gouvernement québécois, expliqua que la campagne financière visait, en premier lieu, à défrayer les coûts des fêtes et, en second lieu, à subventionner la création d'un se- 
crétariat permanent pour la Société nationale l'Assomption. Le comité souligna que le peuple acadien sollicitait un don afin de « mieux jouer un rôle de minorité agressive et [de] mieux soutenir la Province-mère dans sa mission de gardienne officielle de l'héritage français en Amérique ${ }^{63}$ ». Les auteurs du mémoire ne manquèrent pas d'optimisme en affirmant que dans quelques décennies, le Nouveau-Brunswick deviendrait la deuxième province française du Canada. À ce moment, les Acadiens seraient en mesure d'appuyer le Québec « dans ses revendications pour l'avancement du fait français au pays ${ }^{64} »$. Rapidement, le Québec serait en mesure de compter sur l'appui d'une Acadie forte et dynamique dans le combat pour la survie et l'épanouissement du fait français en Amérique.

Le peuple acadien devait donc se servir des fêtes du bicentenaire pour s'élancer vers l'avenir. La force du passé devait l'encourager à viser de plus hauts sommets. Pour cette raison, l'élite en appela à une plus grande participation de l'Acadie à la vie publique, un geste qui présagea peut-être la dynamique des années 1960 et 1970 au Nouveau-Brunswick. Les élites politiques et professionnelles acadiennes furent les porte-parole de ce message. Un député francophone à l'Assemblée législative de la province expliqua que les fêtes illustraient la volonté du peuple acadien de participer davantage au développement du Nouveau-Brunswick «sous toutes ses formes ${ }^{65} \gg$. L'élite souhaita également étendre son champ d'action à la société canadienne. Le temps était venu, selon elle, de revendiquer et d'occuper une juste place sur le plan sociopolitique. Elle voulait démontrer que les Acadiens, ayant atteint l'âge de la maturité, désiraient être « des citoyens utiles à [leur] pays ${ }^{66} »$.

Enfin, l'élite soutenait que la fidélité à l'idéologie traditionnelle, notamment à la foi catholique, à l'histoire et aux traditions, assurait au peuple acadien un avenir prometteur. 
Le discours des fêtes fut, par conséquent, imprégné d'un profond sentiment d'assurance et de confiance en l'avenir. Adélard Savoie déclara que l'esprit de « réveil » qui animait le peuple acadien était le signal d'un nouveau départ, le début d'une période marquant la naissance d'une nouvelle Acadie:

Nos fêtes nous donneront une ardeur rajeunie vers la conquête de nouveaux sommets. Elles marqueront le point de départ d'une ère nouvelle, riche en réalisations insoupçonnées. Il faut nous en rendre compte. Nous commençons une nouvelle étape, celle qui sera le parcours de la véritable montée acadienne ${ }^{67}$.

Calixte Savoie, pour sa part, déclara que " [l]es Aćadiens sont à refaire la conquête de leur Acadie. Ce n'est plus une question de survivance, mais une question de plein épanouissement et de grand rayonnement ${ }^{68} »$. Ces paroles laissèrent entendre qu'il n'était plus question de simple survie pour le peuple acadien. Désormais, ce dernier se fixait de plus nobles objectifs ${ }^{69}$.

\section{$* * *$}

Il appert ainsi que ces célébrations de 1955 ne furent pas annonciatrices d'une nouvelle époque en Acadie. L'idéologie traditionnelle de la survivance était encore bien implantée dans le discours à cette période. L'élite s'y fit le promoteur de l'interprétation historique traditionnelle en rappelant au peuple acadien non seulement l'importance de la Déportation, mais aussi et surtout toute la fierté et la dignité que doit lui inspirer la remontée ultérieure, la Renaissance acadienne, qu'on qualifia de miracle. Elle vit dans la commémoration du bicentenaire une occasion idéale pour instiller un amour et une fierté du passé ancestral aux jeunes et aux moins jeunes. Les fêtes furent imprégnées d'une profonde volonté de vénérer le passé, car le sens de l'histoire et de la tradition devait 
continuer à alimenter les énergies nationales en Acadie. Ainsi, l'élite tenta de projeter la société acadienne vers l'avenir en utilisant le passé comme source d'inspiration.

On ne peut guère être étonné que l'élite acadienne ait conservé sa confiance en l'idéologie traditionnelle de la survivance pour la guider vers le futur. Après tout, celle-ci avait contribué à promouvoir l'avancement du peuple acadien depuis plus d'un siècle. Peut-être certaines élites souhaitaientelles s'éloigner du leitmotiv du passé qui visait essentiellement la survivance acadienne. Toutefois, il ne faudrait pas confondre cette volonté de se tourner avec confiance vers l'avenir avec le début d'une "ère nouvelle ", comme le croit Hautecœur. Les transformations socio-économiques latentes, qui commençaient à s'opérer dans la société acadienne à cette époque, n'étaient pas encore parvenues à ébranler l'idéologie traditionnelle. Il faudra attendre au début des années soixante pour que s'effectue une transition au niveau du discours des élites acadiennes ${ }^{70}$.

\section{NOTES}

' L'auteure tient à remercier le comité de rédaction dont les commentaires ont servi à améliorer le texte. Cette recherche a été réalisée grâce à une Bourse d'études supérieures de l'Ontario et au Fonds d'émergence de projets de recherche sur le Canada français du Centre de recherche en civilisation canadienne-française de l'Université d'Ottawa.

${ }^{2}$ L'imminence d'une guerre entre la France et l'Angleterre au début des années 1750 remit les Acadiens, qui clamaient leur neutralité dans ces conflits impériaux, dans une situation précaire. Charles Lawrence, lieutenant-gouverneur de la Nouvelle-Écosse depuis 1753, estima que les Acadiens menaçaient les intérêts britanniques dans la colonie, surtout parce que ces derniers avaient refusé de prêter serment d'allégeance à la couronne d'Angleterre à maintes reprises. Lawrence donna l'ordre de chasser les Acadiens de la région à l'été 1755, quelques mois avant la déclaration de guerre en Europe. Les chercheurs estiment qu'environ 8000 à 10000 Acadiens furent déportés entre 1755 et 
1763, l'année où le traité de Paris mit fin à la guerre en Europe et en Amérique du Nord, principalement en France et en Grande-Bretagne, en NouvelleAngleterre et en Louisiane. Plusieurs centaines d'Acadiens réussirent à se sauver à l'Île Royale (Île du Cap-Breton), l'Île Saint-Jean (Île-du-PrinceÉdouard) et en Nouvelle-France. Pour de plus amples informations, voir les ouvrages et les articles de John Bartlett Berbner, Andrew Hill Clark, Naomi Griffiths, Émile Lauvrière, Geoffrey Plank, Edme Rameau de Saint-Père et John G. Reid.

3 "Les fêtes au diocèse d'Edmundston », L'Évangéline, 26 juillet 1954.

+ On dénombre dix congrès nationaux entre 1881 et 1937 , qui se déroulèrent à Memramcook (Nouveau-Brunswick) en 1881, à Miscouche (Île-du-PrinceÉdouard) en 1884, à Pointe-de-l'Église (Nouvelle-Écosse) en 1890, à Arichat (N.-É.) en 1900, à Caraquet (N.-B.) en 1905, à Saint-Basile (N.-B.) en 1908, à Tignish (Î.-P.-É.) en 1913, à Pointe-de-l’Église et Grand-Pré (N-.É.) en 1921, à Moncton (N.-B.) en 1927 et à Memramcook (N.-B.) en 1937.

${ }^{5}$ Ces rencontres avaient servi de forums nationaux, les élites acadiennes y discutant de nombreuses questions touchant l'avenir du peuple acadien comme l'éducation, la promotion de la langue et de la religion, l'agriculture, l'émigration et la colonisation. Il existe quelques études portant sur divers aspects des premiers congrès nationaux, par exemple : Ferdinand Robidoux, dir. Les Conventions nationales des Acadiens. Shédiac, Imprimerie du Moniteur acadien, 1907 ; Camille-Antoine Richard. L'idéologie de la première convention nationale. Mémoire de maitrise (sociologie), Université Laval, 1960. 281 p. ; Deborah Robichaud. «Les Conventions nationales (1890-1913) : la Société nationale l'Assomption et son discours ». Les Cabiers de la Société historique acadienne, vol. 12, n" 1 (mars 1981), pp. 36-58. Sheila Andrew a récemment contesté l'interprétation traditionnelle qui fait état de l'unanimité idéologique des élites participant aux congrès nationaux. Voir Sheila Andrew. The Development of Elites in Acadian New Brunswick, 1861-1881. Montréal et Kingston, McGill-Queen's University Press, 1996. 262 p. Gaétan Gervais, auteur d'une étude intéressante sur la participation des Franco-Ontariens aux rassemblements patriotiques canadiens-français de la fin du XIX et du début du XX ${ }^{e}$ siècles, constate que ces congrès remplirent la fonction de "symbole " du Canada français. Voir Gervais. «L'Ontario français et les grands congrès patriotiques canadiens-français (1883-1952)». Cahiers Charlevoix, vol. II (1997), pp. 9-155.

${ }^{6}$ Richard, op. cit., pp. 77-91. L'auteur précise que cette idéologie traditionnelle reposait sur les « grandes traditions » acadiennes, soit le souvenir de la Dépor- 
tation, le culte des ancêtres, la tradition religieuse, la tradition colonisatrice et agricole ainsi que la langue française. Il importe de souligner que le discours idéologique traditionnel subit de légères modifications au fil des ans. La tradition colonisatrice et agricole, qui occupa une place importante dans les discussions des premiers congrès, se vit progressivement reléguer au second plan, pour éventuellement disparaitre entièrement des débats. Le vide laissé fut comblé par les questions d'ordre politique et économique.

${ }^{7}$ On en retrouve des bribes dans quelques ouvrages de synthèse, notamment Robert Rumilly. Histoire des Acadiens. Montréal, Fides, 1955, 2 vol. ; Hugh Thorburn. Politics in New Brunswick. Toronto, University of Toronto Press, 1961. 217 p. ; Roger Ouellette. «Analyse de l'émergence du Parti acadien ». Revue de l'Université de Moncton, vol 16, n" 2 (janvier-mars 1983), pp. 71-88; J.R.H. Wilbur. The Rise of French New Brunswick. Halifax, Formac, 1989. 291 p. ; Jean Daigle, dir. L'Acadie des Maritimes : études thématiques des débuts à nos jours. Moncton, Chaire d'études acadiennes, 1993. 908 p., ainsi que dans certaines autobiographies, notamment Calixte F. Savoie. Mémoires d'un nationaliste acadien. Moncton, Éditions d'Acadie, 1979. 355 p. et Martin J. Légère. Parmi ceux qui vivent: un demi-siècle au service de l'Acadie: mémoires. Moncton, Éditions d'Acadie, 1996. 296 p. Shane Bernard a récemment publié un article portant sur les fêtes régionales du bicentenaire qui eurent lieu en Louisiane. Bernard soutient que l'élite cajun, qui cherchait à obtenir l'approbation de la majorité anglo-saxonne, prépara des fêtes imbues de références à un passé mythique auquel peu de Cajuns s'associaient encore : «Acadian Pride, AngloConformism : the Acadian Bicentennial Celebrations of $1955 \%$. Louisiana History, vol. XLI, n² (printemps 2000), pp. 161-174.

${ }^{8}$ Jean-Paul Hautecœur, L'Acadie du discours, Québec, Les Presses de l'Université Laval, 1975, p. 93. L'élite traditionnelle acadienne réitéra son discours tout en tentant de donner la parole aux novices, à la nouvelle génération d'Acadiens appelés à prendre la relève. Hautecœur conclut que le discours des fêtes révèle une volonté de se tourner vers le futur, mais que ce passage à la modernité devait se faire de façon subtile, car « le nouveau doit côtoyer l'ancien sans que signe de rupture n'apparaisse ».

${ }^{9}$ Philippe Eddie. L'évolution récente du nationalisme chez. les Acadiens du NouveauBrunswick (1955-1980). Mémoire de maîtrise (science politique), Université Laval, 1981.145 p.

${ }^{10}$ Ces chercheurs sont généralement d'avis que la commémoration du patrimoine représente un instrument important dans le processus d'édification de l'État-nation. Dans plusieurs cas, ces études visent à démontrer que l'his- 
toire, et particulièrement les commémorations, fut manipulée par les chefs nationaux à des fins politiques. Sur la commémoration du patrimoine aux États-Unis, voir par exemple les ouvrages de John Bodnar, David Glassberg, Michael Kammen et Patrick Hutton. Ce phénomène se manifeste également au Canada. Dans un ouvrage salué par la critique, H.V. Nelles étudie la commémoration du troisième centenaire de l'arrivée de Champlain à Québec par les gouvernements canadien et québécois. Il constate que cette célébration fut récupérée par les élites à des fins politiques et qu'elle reposait sur la supposition que «l'histoire pouvait créer une nation »: The Art of Nation-Building: Pageantry and Spectacle at Quebec's Tercentenary, Toronto, University of Toronto Press, 1999, p. 11. Robert Cupido arrive à une conclusion semblable dans son étude des fêtes entourant la commémoration du cinquantenaire de la Confédération canadienne : "Appropriating the Past: Pageants, Politics and the Diamond Jubilee of Confederation ». La Revue de la Societé bistorique du Canada / Journal of the Canadian Historical Association, vol. 8 (1998), pp. 155186. Voir l'ouvrage intéressant de Jonathan Vance. Death So Noble: Memory, Meaning and World War One. Vancouver, University of British Columbia Press, 1997. 319 p., qui porte sur la signification des mythes et des rites commémoratifs entourant la Première Guerre mondiale au Canada, ainsi que le chapitre de Ronald Rudin. "Contested Terrain: Commemorative Celebrations and National Identity in Ireland and Quebec ». Dans Yvan Lamonde et Gérard Bouchard, dir., La nation dans tous ses états : le Québec en comparaison, Montréal, Harmattan, 1997, pp. 183-204, qui examine la commémoration au Québec.

"On pense par exemple à Michael Behiels. Prelude to Quebec's Quiet Revolution: Liberalism Versus Neo-Nationalism, 1945-1960. Montréal et Kingston, McGillQueen's University Press, 1985. 366 p. ; Léon Dion. Québec 1945-2000, T.2 Les intellectuels et le temps de Duplessis. Sainte-Foy, Les Presses de l'Université Laval, 1987. 452 p. ; Hubert Guindon, Roberta Hamilton et John L. McMullan, dir. Tradition, modernité et aspiration de la société québécoise. Montréal, Éditions SaintMartin, 1990.233 p.

${ }^{12}$ Marcel Martel, Le deuil d'un pays imaginé : rêves, luttes et déroute du Canada français: les rapports entre le Québec et la francophonie canadienne, 1867-1975, Ottawa, Les Presses de l'Université d'Ottawa, 1997, pp. 75-138.

${ }^{13}$ Simon Langlois, dir. Identité et cultures nationales. Québec, Les Presses de l'Université Laval, 1995. 377 p. Plusieurs études démontrent que des changements importants s'opérèrent en Acadie dans les années soixante. Voir par exemple, Hautecœur, op. cit. ; Louis Cimino. Ethnic Nationalism Among the Acadians of New Brunswick: An Analysis of Ethnic Political Development. Thèse de doctorat (anthropologie), Duke University, 1977. 270 p. ; Lise Ouellette. 
Les luttes étudiantes à l'Université de Moncton: production ou reproduction de la société acadienne. Mémoire de maitrise (sociologie), Université de Montréal, 1982. 128 p. ; Wilbur, op. cit.; Marc Johnson. Les stratégies de l'Acadianité. Analyse socio-historique du rôle de la presse dans la formation de l'identité acadienne. Thèse de doctorat, Université de Bordeaux, 1991.522 p. ; Ricky Richard. I es formes de l'acadianité au Nouveau-Brunswick : action collective et production de l'identité (1960-93). Mémoire de maitrise (science politique), Université Laval, 1994. 123 p. ; J. Yvon Thériault. L'identité à l'épreuve de la modernité. Moncton, Éditions d'Acadie, $1995.323 \mathrm{p}$.

${ }^{14}$ «L'esprit des grands jours du Bicentenaire : la joie », L'Évangéline, 4 août 1955.

${ }^{15}$ On trouvera de l'information concernant la Renaissance acadienne dans les ouvrages suivants : Antoine Bernard. Histoire de la survivance acadienne, 1755 1935. Montréal, Les Clercs de Saint-Viateur, 1935. 465 p. ; Richard. op. cit. ; Raymond Mailhot. La "Renaissance acadienne 》 (1864-1890): linterprétation traditionnelle et Le Moniteur Acadien. Thèse de diplôme en études supérieures, Université de Montréal, 1969. 177 p. et du même auteur Prise de conscience collective acadienne au Nouveau-Brunswick, 1860-1891, et le comportement de la majorité anglophone. Thèse de doctorat (histoire), Université de Montréal, 1973. 486 p.; Andrew, op. cit. Il est également utile de consulter les biographies des personnalités importantes de l'époque. Voir, par exemple, Camille-Antonio Doucet. Une étoile s'est levée en Acadie, Marcel-Francois Richard. CharlesbourgEst, Les Éditions du Renouveau, 1973. 315 p. ; Phyllis Leblanc. « MarcelFrançois Richard ». Dictionnaire biographique du Canada (DBC), vol. XIV, pp. 944-946 ; Étienne Catta. Le Révérend Père Camille Lefebure (1831-1895) et la renaissance acadienne. Moncton, La Province acadienne des Pères de SainteCroix, 1983, 3 vol. ; Naomi Griffiths. « Camille LeFebvre ». DBC, vol. XII, pp. 593-597.

${ }^{16}$ L'Évangéline fut fondée à Digby, Nouvelle-Écosse, par Valentin Landry. En 1905, Landry transporta son entreprise à Moncton, où le journal demeura jusqu'à ce qu'il arrête ses presses en 1982. Pendant ses 95 années d'existence, le journal fut considéré comme l'une des institutions nationales acadiennes. Pour de plus amples renseignements sur l'histoire, le rôle et l'importance du journal pour les communautés acadiennes des Maritimes, le lecteur est prié de consulter l'ouvrage collectif réalisé sous la direction de Gérard Beaulieu. L'Évangéline 1887-1982, entre l'élite et le peuple. Moncton, Éditions d'Acadie et Chaire d'études acadiennes, 1997.416 p. 
${ }^{17}$ Pour une étude détaillée de la Société nationale l'Assomption, consulter R.J. Renault. La Societé nationale des Acadiens. Vol. 2, Moncton, 1973. 221 p. Il faut également faire la distinction entre la Société « nationale » l'Assomption et la Société «mutuelle » l'Assomption. Cette dernière fut fondée à Waltham, Massachusetts, en 1903. Cette organisation fut, au départ, une société d'entraide. Toutefois, quelques années plus tard elle s'engagea dans l'assurancevie. Le siège social de la Société Mutuelle-vie l'Assomption est présentement situé à Moncton. Pour de plus amples informations, consulter Antoine Léger. Les grandes lignes de l'histoire de la Société l'Assomption. Québec, Imprimerie Franciscaine Missionnaire, 1933. 260 p. et Euclide Daigle. Petite histoire d'une grande idée. Moncton, Assomption Compagnie Mutuelle, 1978. 177 p.

${ }^{18}$ Centre d'études acadiennes (CEA), Fonds La Société [mutuelle] l'Assomption (SMA), 77.205 Bicentenaire des Acadiens, "Rapport du comité exécutif », 20 janvier 1950.

${ }^{19}$ À cette fin, la Société entreprit une campagne de financement auprès des paroissiens des diocèses de Moncton, de Bathurst, d'Edmundston et de Yarmouth au mois d'octobre 1954. Le but premier de cette campagne était d'amasser les fonds nécessaires pour mener l'organisation des fêtes à bon port. L'argent recueilli servirait en outre à insuffler un nouveau dynamisme à l'organisme. Euclide Daigle, rédacteur en chef de L'Évangéline, écrivit que les fêtes du bicentenaire mettaient l'importance de la Société nationale l'Assomption en évidence et " [qu']avec la générosité des Acadiens, elles pourraient être l'occasion de la reprise de ses activités ». « Nos deux société l'Assomption », L'Évangéline, 22 octobre 1954.

21" CEA, Fonds SMA, 77.205 Bicentenaire des Acadiens, « Rapport du comité exécutif », 20 janvier 1950.

${ }^{21}$ CEA, Fonds SNA, 41.23-6 Activités: fêtes du bicentenaire, "Causerie de Dr Georges Dumont», s.d. Adélard Savoie décrivit la Déportation comme « un événement si radical et si complet qu'il a bouleversé non seulement l'existence matérielle des Acadiens mais qu'il les a marqués au tréfond[sic] de leur âme d'une empreinte que les siècles n'ont pas effacée. Sans la [D]éportation, les Acadiens ne seraient pas, ne pourraient pas être ce qu'ils sont aujourd'hui ». Pour cette raison, il expliqua que la commémoration du bicentenaire de la Déportation relevait d'un devoir patriotique : «Et si, par indifférence, les chefs de l'Acadie avaient ignoré ou négligé de faire célébrer convenablement le bicentenaire de la Dispersion, n'auraient-ils pas été coupables d'une lâcheté en face de l'histoire, d'un manque de piété filiale à la mémoire des aïeux et d'un manquement dans leurs responsabilités à l'endroit de la nationalité aca- 
dienne ? "CEA, Fonds SNA, 41.23-13 Activités : fêtes du bicentenaire, "Causerie prononcée à Radio-Canada par M. Adélard Savoie », 6 juin 1954.

22 «Le bicentenaire », L'Évangéline, 23 novembre 1953.

${ }^{23}$ CEA, Fonds Bicentenaire des Acadiens, 133.40 Programme, « Programme des fêtes - ébauche ", 1955. Le travail d'organisation des fêtes fut réparti parmi divers comités dont ceux du programme, de la publicité, des publications, des régions, du transport, du logement, des invitations, des finances, des manifestations religieuses, de la reconstitution historique, des décorations, de la parade, du folklore, des concerts, du stationnement, etc. Ces souscomités se reportaient au Secrétariat des Fêtes qui entra en fonction le $1^{\mathrm{er}}$ mars 1954 et qui constituait le centre nerveux de l'organisation.

${ }^{24}$ Le comité organisateur, constatant que les fêtes nationales ne parviendraient qu'à rejoindre une fraction de la population acadienne, décida de diviser le territoire occupé par des Acadiens en cinq grandes régions : les provinces maritimes, le Québec, les États-Unis, l'Ontario et l'Ouest canadien. Dans chaque région s'activèrent des unités locales chargées de conscientiser les gens à l'importance de l'événement et d'organiser des fêtes régionales pour ceux qui ne pourraient se rendre aux célébrations nationales. Adélard Savoie indiqua qu'ainsi, « there will hardly be a region harboring an important group of Acadians that will not be reached by the centennial festivities ». CEA, Fonds SNA, 41.24-7 Fêtes du bicentenaire, "The Maritime Advocate», mai 1955. Pour des descriptions de ces fêtes régionales, voir CEA, Fonds Bicentenaire des Acadiens, 133.43 Bulletins hebdomadaires, 1955 et [Adrien Verrette], «Bicentenaire acadien, 1755-1955 », Bulletin de la Société historique franco-américaine, Nouvelle série, vol. I (1955), pp. 44-49. Le journal L'Évangéline fit également de nombreux reportages sur ces fêtes locales.

${ }^{25}$ Pour assurer aux fêtes tout l'éclat souhaité, le comité organisateur obtint des subventions de plusieurs gouvernements et organisations privées. Les gouvernements de la Nouvelle-Écosse, du Nouveau-Brunswick et du Québec avancèrent respectivement des sommes de $12500 \$, 20000 \$$ et $25000 \$$.

${ }^{26}$ Elle comprenait des cardinaux, le délégué apostolique au Canada, plusieurs évêques et archevêques provenant du Canada, des États-Unis et de la France, l'ambassadeur de la France au Canada, l'ambassadeur des États-Unis, le commissaire du commerce pour la Grande-Bretagne, les ministres fédéraux des Travaux publics et du Travail, plusieurs sénateurs et ministres provinciaux ainsi que le lieutenant-gouverneur du Nouveau-Brunswick : "Nombreux dignitaires à nos fêtes », L'Évangéline, 8 août 1955. 
${ }^{27}$ CEA, Fonds Bicentenaire des Acadiens, 133.40 Programme, « Programmeébauche», s.d.

${ }^{28}$ CEA, Fonds Bicentenaire des Acadiens, 133.37 Programmes, « Programme officiel des fêtes », 1955.

${ }^{29} \mathrm{CEA}$, Fonds SNA, 41.23-6 Activités : fềtes du bicentenaire, "Causerie de M" ${ }^{\text {"r }}$ C.A. LeBlanc», 3 juin 1954.

${ }^{30}$ CEA, Fonds SNA, 41.23-6 Activités: fêtes du bicentenaire, "Discours de Monsieur Adélard Savoie », 1954.

${ }^{31}$ CEA, Fonds Bicentenaire des Acadiens, 133.2 Comité d'organisation, « Procès-verbal de la réunion de l'exécutif de la Société nationale l'Assomption", 28 octobre 1953. Dans le mémoire envoyé au premier ministre du NouveauBrunswick, l'Honorable Hugh John Flemming, décrivant le but, l'organisation, le programme et les besoins financiers du comité des fêtes, le comité organisateur écrivit « We did not want to stir up any sentiments which might endanger in the least our democratic ideal of national unity. Therefore, we are resolutely determined to discard any move or item which might seem objectionable because of the possibility - however remote - that they may foster ill-feeling ». CEA, Fonds SMA, 77.206 Bicentenaire des Acadiens, «Brief respectfully submitted to the Honorable Hugh John Flemming, Premier of the Province of New Brunswick in relation to the Acadian Celebration of $1955 », 16$ décembre 1954, p. 1.

${ }^{32}$ "L'Épiscopat promet appui et participation », L'Évangéline, 26 avril 1954.

${ }^{3}$ À l'Assemblée législative du Nouveau-Brunswick, le futur premier ministre Louis-J. Robichaud prit la parole pour dire à ses collègues que les Acadiens souhaitaient célébrer l'anniversaire d'un événement malheureux mais dont le dénouement n'avait plus rien de tragique. Quelques jours plus tard, André Doucet renchérit sur le fait que les célébrations «ne ressasseraient pas la tragédie de la déportation " mais plutôt le triomphe du peuple acadien dans ses efforts pour maintenir sa religion, sa langue française et ses traditions ». Débats de l'Assemblée législative du Nonveau-Brunswick, 16 mars 1954, p. 187.

${ }^{3+}$ Le Colonel T.L. Bullock, qui aida à l'organisation des fêtes, avait souligné l'importance de bien exécuter cette tâche. Il écrivit à Adélard Savoie au mois de mai 1955, "The message to the Queen from the Acadian people will set the tone for the entire programme, and it must be carefully drawn[...]». CEA, Fonds Bicentenaire des Acadiens, 133.6 Colonel T.L. Bullock, "Correspondance avec Adélard Savoie », 10 mai 1955. 
${ }^{35}$ CEA, Fonds Bicentenaire des Acadiens, 133.34 Message à la Reine, 1955. Neil Pritchard, représentant du haut-commissaire de l'Angleterre aux fêtes, parvint difficilement à dissimuler sa surprise en disant que peu de gens auraient cru voir le jour où les descendants des déportés de 1755 « would extend a warm invitation and a friendly welcome to an official representative of the British government [...]». CEA, Fonds Bicentenaire des Acadiens, 133.33 Discours, sermons, etc., " Discours de Neil Pritchard », 15 août 1955.

${ }^{36} \mathrm{Il}$ importe de souligner que le discours de l'élite fit mention de «pardon» à plusieurs reprises : Calixte Savoie et Adélard Savoie avertirent tous deux que cette commémoration se voulait « une offre généreuse d'un pardon chrétien » car le peuple acadien ne gardait pas de « rancune des souffrances de jadis » et $\mathrm{M}^{\mathrm{gr}}$ Camille LeBlanc, évêque de Bathurst, ajouta que le peuple acadien «fredonn[ait] ensemble le refrain de la chanson acadienne, "Mais pardonnons, c'est Dieu qui nous l'ordonne[...] ». Il serait toutefois erroné de croire que les élites acadiennes pardonnaient à l'Angleterre la Déportation et les injustices du passé à ce moment. Il serait plus juste de voir dans ce geste la promotion d'un esprit de bonne entente nécessaire au caractère chrétien qu'on souhaitait conférer aux célébrations. CEA, Fonds Calixte-Savoie, $64.70 \mathrm{Dis}$ cours, "Bicentenaire de la Déportation des Acadiens ", s.d. ; Fonds SNA, 41.23-6 Activités : fêtes du bicentenaire, «Causerie prononcée à Radio-Canada par M. Adélard Savoie ", le 6 juin 1954 ; Fonds SNA, 41.23-6 Activités : fêtes $d u$ bicentenaire, "Causerie de $\mathrm{M}^{\mathrm{gr}}$ C.A. LeBlanc », 3 juin 1954.

${ }^{37}$ La promotion du discours de bonne entente visait également un objectif plus pragmatique. Le comité des fêtes croyait que les fêtes du bicentenaire fournissaient un excellent prétexte pour exposer et diffuser la notion du dualisme canadien car l'harmonie entre les deux peuples fondateurs au Nouveau-Brunswick se voulait exemplaire de la coopération entre les communautés francophone et anglophone au Canada. Cette intention fut reflétée dans le mémoire envoyé au premier ministre du Nouveau-Brunswick, dans lequel le comité organisateur justifia partiellement sa demande de subvention de 30000 \$ en louangeant le biculturalisme de la province. CEA, Fonds SMA, 77.206 Bicentenaire des Acadiens, «Brief respectfully submitted to the Honorable Hugh John Flemming, Premier of the Province of New Brunswick, in relation to the Acadian Celebration of 1955 », 15 décembre 1954.

${ }^{38}$ Débats de la Chambre des Communes, 17 janvier 1955, p. 260.

${ }^{39}$ CEA, Fonds Bicentenaire des Acadiens, 133.40 Programme, « Programmeébauche», 1955. 
4) CEA, Fonds Calixte-Savoie, 64.73 Bicentenaire des Acadiens, « Message du président ", s.d.

${ }^{41}$ CEA, Fonds Calixte-Savoie, 64.70 Bicentenaire des Acadiens, « Discours : Bicentenaire de la Déportation des Acadiens », s.d.

${ }^{+2}$ CEA, Fonds Bicentenaire des Acadiens, 133.37 Programmes, « Programme officiel des fêtes », 1955. On retrouve également une version commentée de la reconstitution historique dans L'Évangéline du 9 août 1955. Certains chercheurs sont arrivés à la conclusion que l'élite acadienne réaffirma son interprétation de l'histoire acadienne par l'entremise de cette reconstitution historique. Une étude de Judith Perron suggère que par cette reconstitution, l'élite théatralisa son interprétation de l'histoire de l'Acadie, qui repose sur la « mythification du passé engendrée par le discours des rassemblements de la Renaissance ": Théátres, fêtes et célébrations en Acadie, 1880-1980, Université de Moncton, thèse de doctorat, 1995, pp. 111-138. Perron n'est pas seule à croire que les fêtes du bicentenaire véhiculèrent une vision spécifique de l'histoire acadienne. Raymond Pagé, dans son étude comparative sur les reconstitutions historiques de Laurent Tremblay, croit que les Acadiens étaient conviés à une fête "pré-structurée » en 1955. Par la présence privilégiée des élites en place et la nature des événements au programme, il constate que les fêtes du bicentenaire furent conçues par les élites acadiennes pour promouvoir leurs propres intérêts : «L'Acadie en jeu ». Dans Raoul Boudreau et al., dir., Mélanges Marguerite Maillet: recueil de textes de création et d'articles sur la littérature, la langue et l'ethnologie acadiennes en hommage à Marguerite Maillet. Moncton, Les Éditions d'Acadie et Chaire d'études acadiennes, 1996, pp. 271-282.

${ }^{+3}$ "Renseignements variés sur les fêtes du Bicentenaire », L'Évangéline, 5 août 1955. À cette fin, le Comité Central des fêtes et l'Association Acadienne d'Éducation distribuèrent des centaines de drapeaux.

" Évangéline, personnage mythique créé par le poète américain Henry Wadsworth Longfellow en 1847, est devenue la figure mythique du peuple acadien et un véritable symbole national. L'histoire veut qu'une acadienne ait raconté à un Rév. Conolly l'histoire de deux fiancés séparés lors de la Déportation de 1755 à Grand-Pré. La fiancée passa toute sa vie à la recherche de son amoureux et le retrouva, après de nombreuses années, dans un hôpital sur son lit de mort. Le Rév. Conolly raconta à son tour cette histoire d'amour à Longfellow. Le poète trouva l'histoire tellement captivante qu'il entreprit de la conter sous forme de poème, qui fut par la suite traduit en 130 langues. Pour de plus amples informations, consulter le documentaire réalisé par Ginette Pellerin. Évangéline en quête. Montréal, Office national du film, 1995, ainsi que 
l'ouvrage de Robert Viau. Les visages d'Évangéline : du poème au mythe. Beauport, Publications MNH, 1998.190 p.

${ }^{45}$ On voulait également exposer le costume d’Évangéline afin de vendre une certaine image de l'Acadie aux touristes. On prétendait que les femmes habillées en Évangéline plairaient « davantage aux milliers de visiteurs et participants ». «Pendant les fêtes nationales du Bicentenaire », L'Évangéline, 5 août 1955. Le processus de commercialisation du passé fut récemment étudié par Ian McKay. McKay démontre qu'à partir des années 1930, la commémoration du patrimoine en Nouvelle-Écosse fut fortement influencée par le tourisme. Voir « History and the Tourist Gaze: the politics of commemoration in Nova Scotia, 1935-1964 ». Acadiensis, vol. 22, n" 2 (1993), pp. 102-138.

${ }^{46} \mathrm{CEA}$, Fonds Bicentenaire des Acadiens, 133.33 Discours, sermons, etc., " Discours de Willie Belliveau ", 15 août 1955.

${ }^{47}$ Lors d'une réunion qui eut lieu en octobre 1953, M ${ }^{\mathrm{gr}}$ Robichaud déclara qu'il souhaitait que « la note mariale domine les préparatifs et les fêtes ellesmêmes ». CEA, Fonds Bicentenaire des Acadiens, 133.2 Comité d'organisation, «Procès-verbal de la réunion de l'exécutif de la Société nationale l'Assomption », 28 octobre 1953.

${ }^{48} \mathrm{CEA}$, Fonds Bicentenaire des Acadiens, 133.35 Message au Souverain Pontife, «Message de Monseigneur Norbert Robichaud au Souverain Pontife», 1955.

${ }^{49}$ L'élite religieuse ajouta que les Acadiens avaient été récompensés par la Providence pour leur foi et leur fidélité puisque la Déportation, « loin d'avoir été le tombeau de notre petit peuple, est devenu pour lui un glorieux piédestal ». CEA, Fonds SNA, 41.23-6 Activités: fêtes du bicentenaire, «Discours de $\mathrm{M}^{\mathrm{gr}}$ Leménager», 1955.

${ }^{50}$ CEA, Fonds Bicentenaire des Acadiens 133.37, Programmes, « Programme officiel des fêtes ", 1955. Le lendemain, à minuit, on dévoila une statue de l'Assomption en avant de la Cathédrale Notre-Dame de l'Assomption.

${ }^{51}$ CEA, Fonds Bicentenaire des Acadiens 133.12 Colonel T.L. Bullock, « Communiqué de presse », 15 août 1955.

${ }^{52}$ CEA, Fonds SNA, 41.23-6 Activités : fêtes du bicentenaire, « Causerie de $\mathrm{M}^{\mathrm{gr}}$ C.A. LeBlanc», 3 juin 1954.

${ }^{53}$ CEA, Fonds SNA, 41.23-6 Activités : fêtes du bicentenaire, «Causerie de M" ${ }^{\text {gr }}$ Leménager à Radio-Canada », 5 juin 1954.

${ }^{5+}$ CEA, Fonds SNA, 41.23-6 Activités : fêtes du bicentenaire, « Causerie de M" ${ }^{\text {pt }}$ Norbert Robichaud», 25 avril 1954. Il ajouta dans son discours de clôture que 
« les mêmes vertus, le même courage et le même esprit de prière qui a fait la force de nos pères, [seront] notre soutien, notre véritable espoir et notre salut assuré ».

${ }^{55}$ CEA, Fonds Bicentenaire des Acadiens, 133.33 Discours, sermons, etc., " Allocution prononcée au Parc Victoria », 11 août 1955.

56 "Quatre Acadiens seront décorés de l'Ordre de la Fidélité française ", L'Évangéline, 29 novembre 1954. Il s'agissait de $\mathrm{M}^{\mathrm{gr}}$ Norbert Robichaud, archevêque de Moncton, Henri LeBlanc, patriote acadien et archiviste de la Société mutuelle l'Assomption, l'abbé Nazaire Poirier, nationaliste méconnu de la Renaissance et curé en l'̂̂le du Prince-Édouard, et Willie Belliveau, professeur à l'Université Sainte-Anne en Nouvelle-Écosse.

${ }^{57}$ CEA, Fonds SNA, 41.23-6 Activités : fêtes du bicentenaire, «Causerie de $\mathrm{M}^{\text {gr }}$ Leménager», 5 juin 1954. $\mathrm{M}^{\mathrm{vt}}$ Camille LeBlanc expliqua que ces fêtes « fer[aient] surgir inévitablement dans le cœur de la jeunesse Acadienne[sic] des sentiments de fierté nationale avec le désir, l'ambition, la fierté d'imiter leur vaillance[...].CEA, Fonds SNA, 41.23-6 Activités : fêtes du bicentenaire, "Causerie de $\mathrm{M}^{\mathrm{gr}}$ C.A. LeBlanc », 3 juin 1954.

${ }^{58}$ Ces saynètes racontaient une version abrégée de l'histoire traditionnelle acadienne, soit le récit de la Déportation et la période de relève. On lut, par exemple, que Dieu demandait aux Acadiens « de rester fidèles à [leurs] traditions religieuses et nationales ». CEA, Fonds Bicentenaire des Acadiens, 133.32 Saynètes, 1955.

59) CEA, Fonds Calixte-Savoie, 64.68 Bicentenaire des Acadiens, "Discours : L'année 1955 - L’année du souvenir - L'année de l'espérance », 1955. $\mathrm{M}^{\mathrm{gr}}$ Robichaud ajouta à cet effet que les célébrations avaient servi à « raviver la conscience des richesses de notre patrimoine ancestral, la volonté de les faire fructifier et d'en faire profiter avec largesse le prochain.» Voir « Vont-elles avoir un lendemain, ces grandes fêtes? ", L'Évangéline, 17 août 1955.

(6) "Les fêtes du bicentenaire vues par un Madawaskaïen », L'Évangéline, 19 août 1955.

${ }^{61}$ «Puissante montée », L'Évangéline, 8 novembre 1954.

${ }^{62} \mathrm{CEA}$, Fonds Bicentenaire des Acadiens, 133.33 Discours, «Conférence donnée à Radio-Canada par $\mathrm{M}^{\mathrm{gr}}$ Nil Thériault ", 2 octobre 1954. Un éminent Acadien spécifia que l'année du bicentenaire serait « un retour salutaire sur le passé, une récollection, un inventaire de nos sources vives d'où nous pourrons dresser des plans de survivance et de rayonnement ». "Causerie du 
Dr C. Bourque pour l'inauguration du bicentenaire », L'Évangéline, 24 août 1954.

${ }^{63}$ CEA, Fonds SMA, 77.206 Bicentenaire des Acadiens, « Mémoire présenté à l'Honorable Maurice Duplessis, Premier Ministre de la Province de Québec en marge du Bicentenaire de la Déportation des Acadiens », 16 novembre 1954.

${ }^{6+}$ Ibid.

${ }^{65}$ "Hommage aux Acadiens par deux députés de l'Assemblée législative ", L'Évangéline, 19 février 1954.

${ }^{60}$ "Les fêtes du deuxième centenaire», Le Madawaska, 29 juillet 1954. Hervé Michaud ajouta dans un discours à la Chambre des communes que le peuple acadien désirait contribuer à l'expansion culturelle, économique et morale du pays. Débats de la Chambre des communes, 3 mai 1954, p. 4603.

${ }^{67}$ CEA, Fonds Calixte-Savoie, 64.68 Bicentenaire des Acadiens, " Discours : L'année 1955 - L'année du souvenir - L'année de l'espérance», 1955.

${ }^{68}$ CEA, Fonds SNA, 41.23-13, Activités: fêtes du bicentenaire, "Conférence donnée au Club Richelieu de Montréal », 13 janvier 1955.

(9) On jugea donc important de doter l'Acadie de moyens nécessaires pour atteindre ces objectifs. Le congrès de 1955 donna l'impulsion qui mena, deux ans plus tard, lors du douzième congrès national des Acadiens tenu à Memramcook en 1957, à la création d'un secrétariat permanent à la Société nationale l'Assomption. Le secrétariat, qu'on comparait au Conseil de la Vie française en Amérique, allait être au service de tous les organismes acadiens. Il servirait de « cerveau ou [de] centre nerveux [à] l'Acadie bicentenaire » puisqu'il serait chargé de coordonner les nombreuses activités, de canaliser les énergies et de déterminer les grands projets. Le secrétariat serait au service de l'Acadie, mais il servirait également de défenseur des minorités françaises en Amérique en assurant leur appui et leur support. Cette idée plut tellement aux dirigeants du Conseil de la vie française que ceux-ci versèrent $25000 \$$ pour relancer la Société et la doter d'un secrétariat permanent. Le premier bureau permanent de la Société nationale des Acadiens ouvrit ses portes au mois d'août 1958 à Moncton. Lors du congrès de Memramcook, les participants proposèrent également un projet de rajeunissement de l'organisation. Les élites exprimèrent le désir de doter la communauté d'un organisme dynamique qui œuvrerait avec compétence dans les diverses sphères d'activités acadiennes. À ce moment, la Société nationale l'Assomption devint la Société nationale des Acadiens. Pour de plus amples renseignements sur ce congrès, voir «La Société nationale des Acadiens - historique », Nos forces vives face à 
l'avenir: XIV" congrès général des Acadiens, Caraquet, Société nationale des Acadiens, 1965, pp. 13-14

${ }^{70}$ L'élite acadienne dut remettre en question la viabilité de l'idéologie traditionnelle lors du congrès national de 1960 qui gravita autour des thèmes de l'évolution et de l'adaptation. Le congrès, qui eut lieu à Pointe-de-l'Église en Nouvelle-Écosse, visait à déterminer les besoins du peuple acadien. Adressant la parole aux participants du congrès, le Père Clément Cormier explicita le défi de l'heure. "Nous allons nous demander si nos concepts patriotiques, nos méthodes d'action, notre programme économique, nos aspirations culturelles ne sont pas trop servilement inspirés des idées qui ont été élaborées et promulguées en un autre siècle. Nous aurons à déterminer si par fidélité nous devons continuer d'adhérer à ces mêmes idées, ou si par souci de progrès nous devons les abandonner » : CEA, Fonds SNA, 41.6-14 Pointe-del'Église, 1960, «Introduction aux séances d’étude », 12 août 1960. 